\title{
Extraction of "hidden" relaxation times from AC susceptibility data
}

\author{
Daniel Reta and Nicholas F. Chilton* \\ Department of Chemistry, School of Natural Sciences, The University of Manchester, \\ Oxford Rd, M13 9PL, U.K.
}

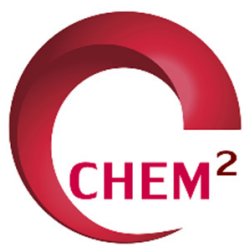

Email: nicholas.chilton@manchester.ac.uk

\begin{abstract}
Magnetic relaxation times are the central observable in defining the characteristics of singlemolecule magnets. It is not uncommon to observe multiple distinct relaxation timescales for a pure material, and sometimes only one relaxation timescale is observed in samples containing multiple components. Herein we examine the conditions required for two distinct relaxation processes to be observable in alternating current susceptibility experiments. We find that there must be at least one order of magnitude difference in the two relaxation times $\left|\log _{10}\left[\tau_{\mu, 1} / \tau_{\mu, 2}\right]\right|>0.922$, even when the intrinsic distributions of the two processes are infinitely sharp $(\alpha=0$ in the generalised Debye model). In the case where only one process is observable, we provide an expression to estimate the two underlying relaxation times, allowing extraction of "hidden" information from AC susceptibility data.
\end{abstract}

Keywords: Magnetic relaxation, AC susceptibility, Debye model.

\section{Introduction}

Molecular magnets have fascinated chemists and physicists alike for the best part of a century, because their structure, dimensionality and physical properties can be tuned via synthesis. These materials can be zero-, one-, two- or three-dimensional magnetic systems that can serve as textbook examples of finite or extended quantum systems [1]. Single-molecule magnets (SMMs) have been of particular interest over the last 30 years: these are zero-dimensional molecular magnets (i.e. isolated molecules) that show slow magnetic dynamics intrinsic to the molecular unit [2]. SMMs are characterised by their magnetic relaxation time $\tau_{\mu}$ (or reciprocally the relaxation rate $\tau_{\mu}{ }^{-1}$ ), which is related to the half-life of the samples return to magnetic equilibrium as $t_{1 / 2}=\tau_{\mu} \ln [2]$. The temperature and magnetic field dependence of $\tau_{\mu}$ gives insight into the underlying spin-phonon coupling mechanisms responsible for reequilibration [3-5]. Magnetic relaxation is most commonly measured by alternating current (AC) susceptometry [6] but also often by direct current (DC) magnetisation decay when the relaxation time falls below the AC frequency window. Both techniques often show evidence of distributions in the relaxation time ( $\alpha$ and $\beta$ parameters in the generalised Debye (AC) and stretched exponential (DC) model functions, respectively [2]). In many SMMs, these distributions are far broader than the experimental error in measuring $\tau_{\mu}$, and thus we have recently developed a method for obtaining estimated standard deviations (ESDs) for the relaxation times as measured by AC [7] and DC [8] methods. Here we now turn to the problem of multiple relaxation times, which is a relatively common observation in SMMs [9-15]. The origin of multiple relaxation times is often associated with inequivalent magnetic sites in a material, however there are many reports where multiple relaxation times are observed where only one crystallographically unique site is present, and indeed this can be explained either by the underlying electronic structure (i.e. presence of multiple electronic states through which relaxation can proceed, and hence multiple relaxation processes) [16], or to a broad distribution of intermolecular dipolar magnetic fields leading to different local magnetic fields [17]. However, the physical origin or interpretation of multiple relaxation processes is not the focus of this work, and rather we are interested in the conditions for observation, or not, of two relaxation times $\left(\tau_{\mu, 1}\right.$ and $\left.\tau_{\mu, 2}\right)$ in AC susceptibility 
experiments. By comparing the magnetic susceptibility profiles of the generalised Debye model for single relaxation times with those of a generalised Debye model with two distinct relaxation times, we find that the out-of-phase AC susceptibility will appear as a single peak when $\left|\log _{10}\left[\tau_{\mu, 1} / \tau_{\mu, 2}\right]\right|<0.580$, and will resolve into two clear peaks when $\left|\log _{10}\left[\tau_{\mu, 1} / \tau_{\mu, 2}\right]\right|>0.922$, even for infinitely sharp distributions (i.e. $\alpha=0)$. In the case where only a single peak is observed $\left(\left|\log _{10}\left[\tau_{\mu, 1} / \tau_{\mu, 2}\right]\right|<0.580\right)$, we also derive an expression to estimate the two underlying relaxation times, allowing extraction of "hidden" information from AC susceptibility data.

As a case study, we examine the recently-described SMM $\left[\mathrm{Dy}(\mathrm{OPh})_{2}(\mathrm{py})_{5}\right]\left[\mathrm{B}(\mathrm{Ph})_{4}\right]$ [18] where two relaxation times are observed in the $\mathrm{AC}$ data between 30 and $40 \mathrm{~K}$, and only one relaxation time above $40 \mathrm{~K}$. However, the alpha values between 40 and $50 \mathrm{~K}$ are abnormally large $(0.05<\alpha<0.15$ cf. $\alpha<$ 0.03 at other temperatures), suggesting that two processes are obscured in this range. Using our method we are able to extract the two underlying relaxation times in this temperature range and show that they smoothly converge to a single high-temperature relaxation process, rather than one process abruptly switching off or on.

\section{Model.}

For a material with a single magnetic relaxation time, the (real) in- and (imaginary) out-of-phase AC susceptibility components are well described by the generalised Debye model (herein the "single generalised Debye" model or SGD) [6], Equations 1-3, respectively [2]. Here, $\tau_{\mu}$ is the characteristic relaxation time, $\alpha$ is the distribution parameter, $\omega$ is the AC frequency and $\chi_{S}$ and $\chi_{T}$ are the adiabatic and isothermal susceptibilities, respectively. Subsequently, we consider a material with two components of equal proportion, only differing in their characteristic relaxation times (i.e. $\chi_{S, 1}=\chi_{S, 2}=\chi_{S}, \chi_{T, 1}=$ $\chi_{T, 2}=\chi_{T}, \alpha_{1}=\alpha_{2}=\alpha$; the more general case is discussed at the end of the manuscript), and for simplicity we define $\tau_{\mu, 1}=10^{-s}$ and $\tau_{\mu, 2}=10^{s}$, so that a single parameter $s$ controls the separation of the two species symmetrically on a logarithmic scale about $\tau=1$; in this case the AC susceptibilities are given by Equations 4-6 (herein the "double generalised Debye" model or DGD; note the factor of 1/2 in Equation 4, that also propagates into Equations 5 and 6, differs from the common definition [10], and is employed here such that when the two processes occur on the timescale $\tau_{\mu, 1}=\tau_{\mu, 2}$ the equation is the same as the SGD).

$$
\begin{gathered}
\chi_{S G D}(\omega)=\chi_{S}+\frac{\chi_{T}-\chi_{S}}{1+\left(i \omega \tau_{\mu}\right)^{1-\alpha}} \\
\chi_{S G D}^{\prime}(\omega)=\chi_{S}+\left(\chi_{T}-\chi_{S}\right) \frac{1+\left(\omega \tau_{\mu}\right)^{1-\alpha} \sin [\pi \alpha / 2]}{1+2\left(\omega \tau_{\mu}\right)^{1-\alpha} \sin [\pi \alpha / 2]+\left(\omega \tau_{\mu}\right)^{2-2 \alpha}} \\
\chi_{S G D}^{\prime \prime}(\omega)=\left(\chi_{T}-\chi_{S}\right) \frac{\left(\omega \tau_{\mu}\right)^{1-\alpha} \cos [\pi \alpha / 2]}{1+2\left(\omega \tau_{\mu}\right)^{1-\alpha} \sin [\pi \alpha / 2]+\left(\omega \tau_{\mu}\right)^{2-2 \alpha}} \\
\chi_{D G D}(\omega)=\frac{1}{2}\left(2 \chi_{S}+\frac{\chi_{T}-\chi_{S}}{1+\left(i \omega 10^{-s}\right)^{1-\alpha}}+\frac{\chi_{T}-\chi_{S}}{1+\left(i \omega 10^{s}\right)^{1-\alpha}}\right)
\end{gathered}
$$




$$
\begin{gathered}
\chi_{D G D}^{\prime}(\omega)=\chi_{S}+\frac{1}{2}\left(\chi_{T}-\chi_{S}\right)\left(\begin{array}{c}
\frac{1+\left(\omega \tau_{\mu, 1}\right)^{1-\alpha} \sin [\pi \alpha / 2]}{1+2\left(\omega \tau_{\mu, 1}\right)^{1-\alpha} \sin [\pi \alpha / 2]+\left(\omega \tau_{\mu, 1}\right)^{2-2 \alpha}} \\
+\frac{1+\left(\omega \tau_{\mu, 2}\right)^{1-\alpha} \sin [\pi \alpha / 2]}{1+2\left(\omega \tau_{\mu, 2}\right)^{1-\alpha} \sin [\pi \alpha / 2]+\left(\omega \tau_{\mu, 2}\right)^{2-2 \alpha}}
\end{array}\right) \\
\chi_{D G D}^{\prime \prime}(\omega)=\frac{1}{2}\left(\chi_{T}-\chi_{S}\right)\left(\begin{array}{c}
\frac{\left(\omega \tau_{\mu, 1}\right)^{1-\alpha} \cos [\pi \alpha / 2]}{1+2\left(\omega \tau_{\mu, 1}\right)^{1-\alpha} \sin [\pi \alpha / 2]+\left(\omega \tau_{\mu, 1}\right)^{2-2 \alpha}} \\
+\frac{\left(\omega \tau_{\mu, 2}\right)^{1-\alpha} \cos [\pi \alpha / 2]}{1+2\left(\omega \tau_{\mu, 2}\right)^{1-\alpha} \sin [\pi \alpha / 2]+\left(\omega \tau_{\mu, 2}\right)^{2-2 \alpha}}
\end{array}\right)
\end{gathered}
$$

\section{Results and Discussion}

The first question that we ask is what combination of separation $s$ and broadening $\alpha$ is required to observe two distinct peaks in the out-of-phase AC susceptibility of the DGD model. Clearly, for large separations and narrow distributions (small $\alpha$ ) two peaks can be observed (Figure 1, black), however when the separation is smaller (Figure 1, red) or the distributions are wider (Figure 1, blue), only one peak is observed. We can define the visibility of two maxima $(V)$ with Equation 7 , as the difference between the $\chi^{\prime \prime}$ values at $\omega=\tau_{\mu, 1}{ }^{-1}=10^{s}$ (one of the maxima) and $\omega=1$ (the central minimum, due to the definition of $\tau_{\mu, 1}$ and $\tau_{\mu, 2}$ ); note that we could equivalently define the visibility with $\omega=\tau_{\mu, 2}{ }^{-1}=$ $10^{-s}$ instead. When $V<0$ only a single peak can be observed and when $V>0$ two peaks can be observed; thus, $V=0$ defines the "line of visibility" (here we exclude the trivial case of $s=0$ for which $\mathrm{V}$ is always equal to zero). In the limiting case of $\alpha=0, s \approx 0.461$ for $V=0$, and when the intrinsic broadening becomes larger there must be a larger separation in order to observe two peaks: e.g. when $\alpha=0.2,0.4,0.6$ or $0.8, s \approx 0.665,0.982,1.58$ or 3.28 , respectively, for $V=0$, Figure 2 (circles). In the limit $\alpha \rightarrow 1, \chi^{\prime \prime} \rightarrow 0$ for all frequencies and thus $s$ diverges.

$$
\begin{aligned}
& V=\chi_{D G D}^{\prime \prime}\left(10^{s}\right)-\chi_{D G D}^{\prime \prime}(1)= \\
& =\frac{1}{2}\left(\chi_{T}\right. \\
& \left.-\chi_{S}\right) \cos [\pi \alpha / 2]\left(\left(\begin{array}{c}
\frac{1}{2+2 \sin [\pi \alpha / 2]} \\
+\frac{10^{2 s(1-\alpha)}}{1+2 \times 10^{2 s(1-\alpha)} \sin [\pi \alpha / 2]+10^{4 s(1-\alpha)}}
\end{array}\right)\right. \\
& \left.-\left(\begin{array}{c}
\frac{10^{-s(1-\alpha)}}{1+2 \times 10^{-s(1-\alpha)} \sin [\pi \alpha / 2]+10^{-2 s(1-\alpha)}} \\
+\frac{10^{s(1-\alpha)}}{1+2 \times 10^{s(1-\alpha)} \sin [\pi \alpha / 2]+10^{2 s(1-\alpha)}}
\end{array}\right)\right)
\end{aligned}
$$


a)

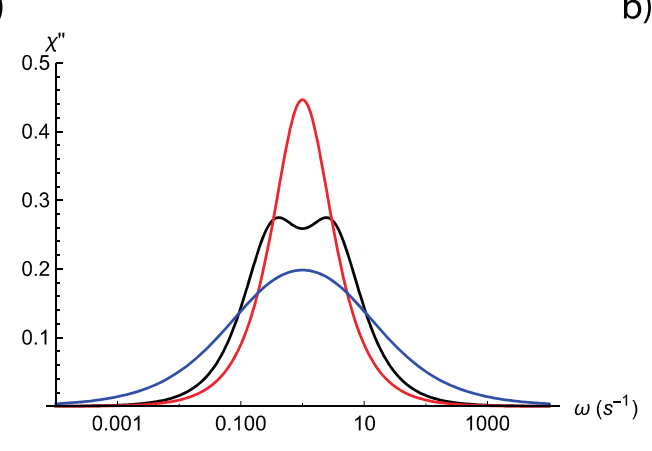

b)

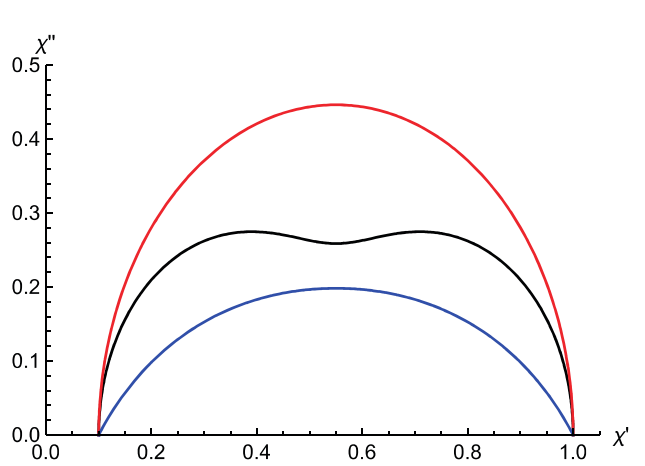

Figure 1. Out-of-phase AC susceptibility (a) and Cole-Cole plots (b) for DGD models with parameters $s=0.5$ and $\alpha=0.001$ (black), $s=0.05$ and $\alpha=0.001$ (red), and $s=0.5$ and $\alpha=0.4$ (blue). $\chi_{s}=$ $0.1, \chi_{T}=1, \tau_{\mu, 1}=10^{-s}$ and $\tau_{\mu, 2}=10^{s}$ in all cases.

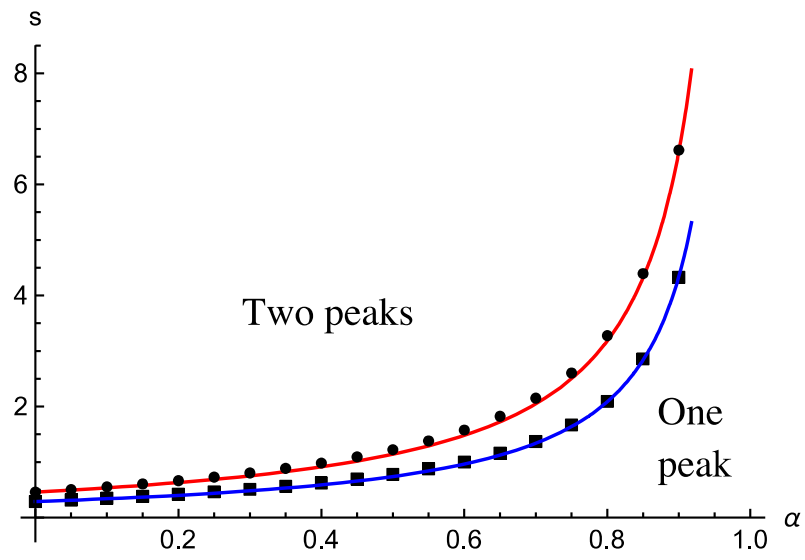

Figure 2. Lines of visibility. Above the red line two peaks can be observed, and below the blue line only a single peak can be observed, by our two different definitions. Circles are solutions to Equation 7 for $V=0$, red line is best fit with Equation 8 where $C_{1}=0.461$ and $C_{2}=0.681$. Squares are $S_{\text {max }}$ values for SGD fits to DGD data, having enforced $\mathrm{R}^{2}=0.998$, and blue line is best fit with Equation 8 where

$$
C_{1}=0.290 \text { and } C_{2}=0.450 \text {. }
$$

The line of visibility (Equation 7,V=0) is very well approximated by Equation 8, where $C_{1}=0.461$ is fixed by the $\alpha=0$ intercept and the best-fit for $C_{2}=0.681$, Figure 2 (red line). This line defines the maximum value of the separation $s_{\max }$ that is possible for a given value of $\alpha$ such that only one peak is observed. However, the shape of the out-of-phase peak for the DGD model near this line of visibility will deviate greatly from that predicted by the SGD model, even if there is technically only one peak, e.g. Figure S1. Hence, a more practical definition of the line of visibility may be one based on the goodnessof-fit of a SGD model to the $\chi^{\prime \prime}$ trace of a DGD dataset. In this approach, the SGD model is parameterised by an effective broadening $\alpha_{e f f}$ (with $\tau_{\mu, e f f}=1$ due to our definition of $\tau_{\mu, 1}=10^{-s}$ and $\tau_{\mu, 2}=10^{s}$ ) and we fit the data from a DGD model of given $\alpha$ and $s$; this means that $\alpha_{e f f}>\alpha$ due to the convolution of two process with distinct relaxation times. Here we choose a reduced $\mathrm{R}^{2}$ coefficient of 0.998 as our cut-off for "a good fit" by visual inspection (see Figure S2, where the parameters are now different to that in Figure $\mathbf{S 1}$ and hence the data fit better to a SGD model), and thus we can define more practical values for $s_{\max }{ }^{\prime}$, Figure 2 (squares). These data are also very-well approximated by Equation 8, where again $C_{1}=0.290$ is fixed by the $\alpha=0$ intercept and the best-for for $C_{2}=0.450$, Figure 2 (blue line). As the $\alpha=0$ intercept (representing infinitely sharp distributions) has a non-zero value of $s_{\max }$ even for our more stringent $\mathrm{R}^{2}$ definition of $s_{\max }{ }^{\prime}$, a difference of up to half an order of magnitude (blue line in Figure $2,\left|\log _{10}\left[\tau_{\mu, 1}\right]-\log _{10}\left[\tau_{\mu, 2}\right]\right|=2 s_{\text {max }}{ }^{\prime} \approx 0.580$ ) 
between two relaxation times will still resemble a single peak with a larger effective broadening $\alpha_{\text {eff }}$. When the separation is increased for $\alpha=0$ (i.e. moving upwards along the $s$-axis in Figure 2), separations around an order of magnitude (red line in Figure $2,2 s_{\max } \approx 0.922$ ) and greater will give rise to two clear peaks in the out-of-phase susceptibility. Both separation bounds will be larger when the intrinsic broadening $\alpha$ of the two processes increases.

$$
s_{\max }=C_{1}+\frac{C_{2} \alpha}{1-\alpha}
$$

For values of $0<s<s_{\max }{ }^{\prime}$ the out-of-phase peak of the DGD function is well-modelled by a SGD model with $\alpha_{e f f}>\alpha$. When $s=0$ the two relaxation times are identical and hence $\alpha_{e f f}=\alpha$, but as $s$ increases for fixed $\alpha$ there is a concomitant increase in $\alpha$ eff , Figure 3. Interestingly, the contour lines of iso- $\alpha$ appear to be quadratic functions of $s$ with flattening profiles upon increasing $\alpha$; this is consistent with the limit $\alpha_{\text {eff }}=1$ when $\alpha=1$, because no peaks are observed and $s$ could take any value, and thus the $\alpha=1$ contour is a flat line at $\alpha_{e f f}=1$. By fitting these data with quadratic functions $\alpha_{e f f}=$ $A_{a} s^{2}+\alpha$, Figure S3, we can determine how the quadratic coefficient varies with $\alpha$ (where we can define $A_{a}=0$ for $\alpha=1$ ), Figure $\mathbf{S} 4$. The values of $A_{a}$ as a function of $\alpha$ are very well approximated by $A_{a}=1.33(1-\alpha) e^{-2.82 \alpha}$, Figure $\mathbf{S} 4$, allowing us to approximate how $\alpha_{\text {eff }}$ varies with $\alpha$ and $s$, Equation 9.

$$
\alpha_{e f f}=1.33(1-\alpha) e^{-2.82 \alpha} s^{2}+\alpha
$$

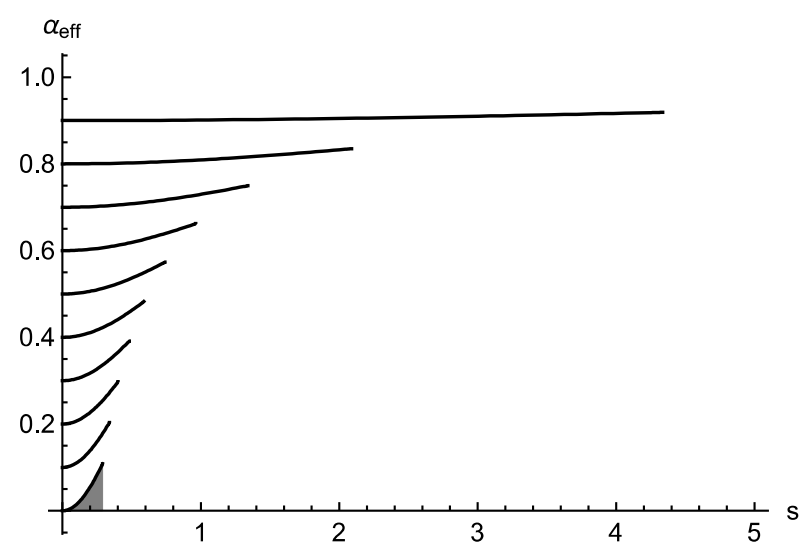

Figure 3. Contours of the effective distribution parameter for a SGD model describing an underlying bimodal distribution given by the DGD model. Each contour intersects the vertical axis at its value of iso- $\alpha$. The gray-shaded region is not permissible parameter space because even when $\alpha=0$, $\alpha_{\text {eff }}=1.33 s^{2}$ in the range $0<s \lesssim 0.290$.

With our collaborators we have recently reported a series of high-performance pentagonal bipyramidal Dy(III) SMMs, where $\left[\mathrm{Dy}(\mathrm{OPh})_{2}(\mathrm{py})_{5}\right]\left[\mathrm{B}(\mathrm{Ph})_{4}\right]$ showed two relaxation times in the AC data between 30 and $40 \mathrm{~K}$ (only half the second peak is observed at 26 and $28 \mathrm{~K}$ ), and just one relaxation time between 20 and $24 \mathrm{~K}$ and above $40 \mathrm{~K}$ [18]. However, before two peaks emerge upon cooling, there is a dramatic increase in $\alpha_{\text {eff }}$ for the single observed relaxation peak, Figure 4. Under the assumptions of our model above, if the intrinsic $\alpha$ parameters of the two underlying relaxation process are similar enough (with respect to a significantly larger $\alpha_{e f f}$ ), then we can approximate the separation of the two peaks in the data assuming they are symmetrically disposed around the measured $\tau_{\mu, e f f}$. Given the values of $\alpha_{1}$ and $\alpha_{2}$ are $c a .0 .009$ for $30 \mathrm{~K} \leq \mathrm{T} \leq 40 \mathrm{~K}$, and return to $c a .0 .014$ for $\mathrm{T}>50 \mathrm{~K}$, we assume an intrinsic average value of $\alpha=0.012$ for the data in the range $46 \mathrm{~K} \leq \mathrm{T} \leq 50 \mathrm{~K}$ (we note that the decrease of 
$\alpha_{\text {eff }}$ at 42 and $44 \mathrm{~K}$ is due to the divergence of the two processes, but there is not enough lowfrequency data to model the slower relaxation process; the same is true for data below $30 \mathrm{~K}$ ). Rearranging Equation 9 to find the separation $s$, we obtain Equation 10 and thus with our value of $\alpha=$ 0.012 we can find $s$ for each value of $\alpha_{\text {eff }}$ in the range $46 \mathrm{~K} \leq \mathrm{T} \leq 50 \mathrm{~K}$. With the relation that $\tau_{\mu, 1}=10^{-s} \tau_{\mu, \text { eff }}$ and $\tau_{\mu, 2}=10^{s} \tau_{\mu, \text { eff }}$, we can extract approximate relaxation times for the two underlying processes, Figure 5. This suggests that the two process indeed converge to a single relaxation time at higher temperatures, which was not visible in the original treatment of the data [18]. The relaxation rate data for the fast $\left(\tau_{\mu, 1}\right)$ and slow $\left(\tau_{\mu, 2}\right)$ relaxation processes can now be fitted (Figure 5) with Equation 11 using CC-FIT2 [7] to obtain the model parameters, Table 1.

$$
\begin{gathered}
s \approx \sqrt{\frac{\alpha_{e f f^{-\alpha}}}{1.33(1-\alpha) e^{-2.82 \alpha}}} \\
\log _{10}\left[\tau_{\mu, n}{ }^{-1}\right]=\log _{10}\left[10^{-A} e^{-U_{e f f} / T}+10^{R} T^{n}\right]
\end{gathered}
$$

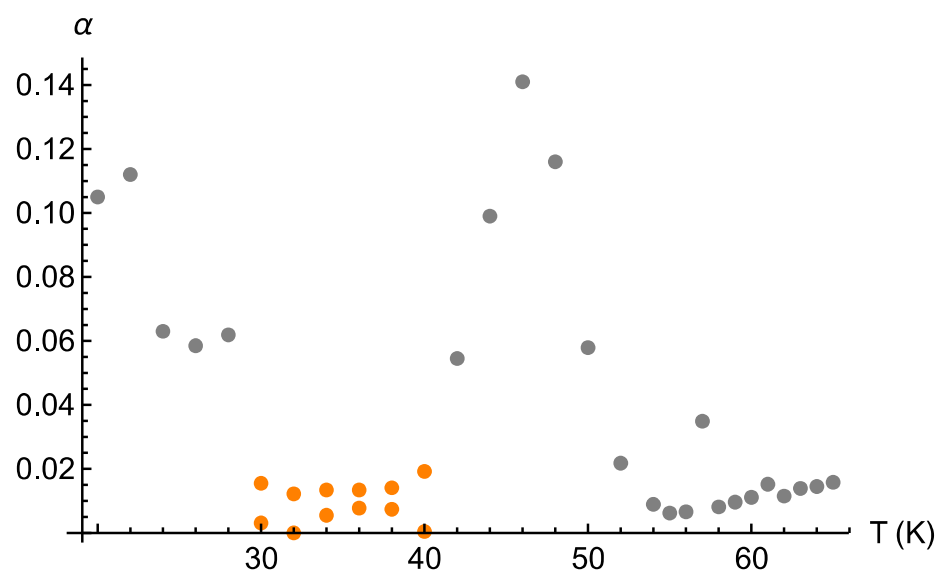

Figure 4. Values of $\alpha_{1}$ and $\alpha_{2}$ (orange points, $30 \mathrm{~K} \leq \mathrm{T} \leq 40 \mathrm{~K}$, DGD fits), and $\alpha_{\text {eff }}$ (grey points, $\mathrm{T}<30 \mathrm{~K}$ and $\mathrm{T}>40 \mathrm{~K}$, SGD fits) for $\left[\mathrm{Dy}(\mathrm{OPh})_{2}(\mathrm{py})_{5}\right]\left[\mathrm{B}(\mathrm{Ph})_{4}\right][18]$.

Table 1. Model relaxation parameters

\begin{tabular}{ccccc}
\hline Process & $\boldsymbol{U}_{\boldsymbol{e f f}}(\mathbf{K})$ & $\boldsymbol{A}(\log [\mathbf{s}])$ & $\boldsymbol{R}\left(\log \left[\mathbf{s}^{-1} \mathbf{K}^{-n}\right]\right)$ & $\boldsymbol{n}$ \\
\hline Fast, $\boldsymbol{\tau}_{\boldsymbol{\mu , 1}}$ & $1320(80)$ & $-12.6(6)$ & $-5.9(5)$ & $4.5(3)$ \\
\hline Slow, $\boldsymbol{\tau}_{\boldsymbol{\mu , \boldsymbol { 2 }}}$ & $1130(50)$ & $-11.3(3)$ & $-7.6(9)$ & $5.0(6)$ \\
\hline
\end{tabular}




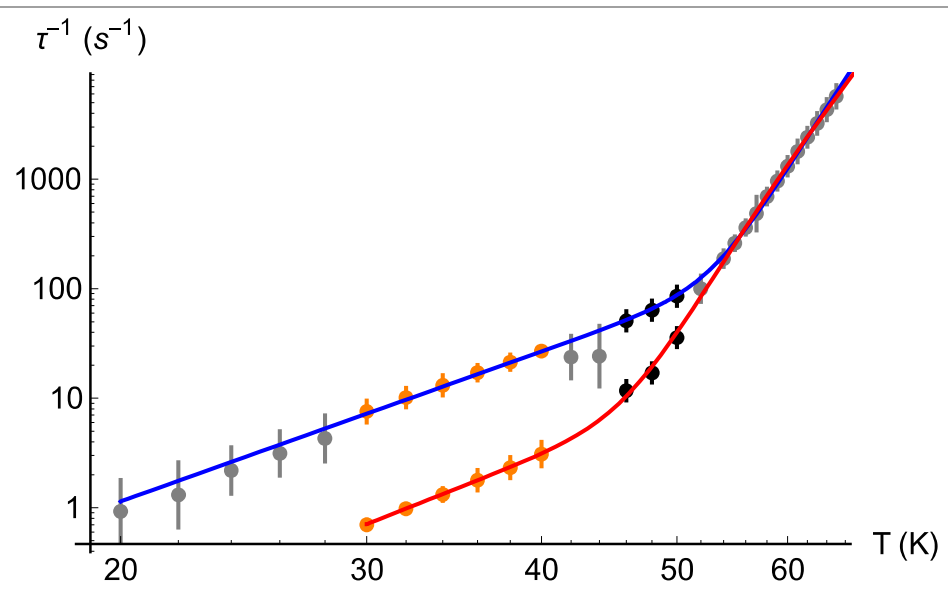

Figure 5. Temperature dependence of the magnetic relaxation rates for $\left[\mathrm{Dy}(\mathrm{OPh})_{2}(\mathrm{py})_{5}\right]\left[\mathrm{B}(\mathrm{Ph})_{4}\right][18]$. Data are from the DGD model (orange), the SGD model (grey), and extracted using the method derived herein (black). Error bars are ESDs from the log-normal distribution model according to ref. 7. Blue line is a fit to the faster relaxation data $\tau_{\mu, 1}$ (grey points above $50 \mathrm{~K}$, below $30 \mathrm{~K}$ and at 42 and $44 \mathrm{~K}$; upper black points; upper orange points), red line is a fit to the slower relaxation data $\tau_{\mu, 2}$ (grey points above 50 $\mathrm{K}$; lower black points; lower orange points).

Our final point of discussion is to address the situation where the susceptibilities of the two components are not identical. It has previously been discussed that the magnitudes of the magnetic susceptibilities of the two relaxing species must not differ too largely in order for two processes to be observable [16]. Performing simulations with variable ratios of $\left(\chi_{T, 1}-\chi_{S, 1}\right) /\left(\chi_{T, 2}-\chi_{S, 2}\right)$ (Figure S5) shows that $\left(\chi_{T, 1}-\chi_{S, 1}\right) /\left(\chi_{T, 2}-\chi_{S, 2}\right)$ should lie between 0.1 and 10 for two processes to be observable, and that two processes are more easily observed the closer this ratio is to unity. Indeed, in refs 9, 11 and 12 the ratio of $\left(\chi_{T, 1}-\chi_{S, 1}\right) /\left(\chi_{T, 2}-\chi_{S, 2}\right)$ is in the range $0.3-3.6$, in agreement with the above conditions. Generalisation of our methodology to more complex cases with $\chi_{S, 1} \neq \chi_{S, 2}, \chi_{T, 1} \neq \chi_{T, 2}, \alpha_{1} \neq \alpha_{2}$ is not trivial and would not be advisable; extraction of "hidden" relaxation data should be done with only the simplest possible models, as variation amongst the other parameters would lead to arbitrary solutions.

\section{Conclusions}

We have examined the conditions, accounting for separation and breadth of the intrinsic distributions, under which two distinct magnetic relaxation processes will be observable in AC susceptibility experiments. We find a minimum separation of approximately an order of magnitude is required to observe two relaxation processes, which increases when the intrinsic distributions are broader. We have also examined the case where only a single process is observed, but is suspected to arise from two underlying processes, and derived an expression relating the effective distribution of the single process to the separation of the two underlying processes, allowing extraction of "hidden" information from AC susceptibility data.

\section{Conflict of Interests}

The authors declare there is no conflict of interests. 


\section{Acknowledgements}

We thank The Royal Society (University Research Fellowship to NFC) and The University of Manchester for support. This project has received funding from the European Research Council (ERC) under the European Union's Horizon 2020 research and innovation programme (grant agreement No. 851504). We thank Dr. Michael Baker for helpful comments.

\section{References}

[1] Gao, S., (Ed.), Molecular Nanomagnets and Related Phenomena, Springer Berlin Heidelberg, Berlin, Heidelberg, 2015, vol. 164.

[2] Gatteschi, D.; Sessoli, R.; Villain, J., Molecular Nanomagnets, Oxford University Press, 2006.

[3] Abragam, A.; Bleaney, B., Electron Paramagnetic Resonance of Transition Ions, Oxford University Press, 1970.

[4] Ding, Y.-S.; Yu, K.-X.; Reta, D.; Ortu, F.; Winpenny, R. E. P.; Zheng, Y.-Z.; Chilton, N. F., Nat. Commun., 2018, 9, 3134.

[5] Escalera-Moreno, L.; Baldoví, J. J.; Gaita-Ariño, A.; Coronado, E., Chem. Sci., 2018, 9, $3265-3275$.

[6] Topping, C. V.; Blundell, S. J., J. Phys. Condens. Matter, 2019, 31, 013001.

[7] Reta, D.; Chilton, N. F., Phys. Chem. Chem. Phys., 2019, 21, 23567-23575.

[8] Evans, P.; Reta, D.; Whitehead, G. F. S.; Chilton, N. F.; Mills, D. P., J. Am. Chem. Soc., 2019, 141, 19935-19940.

[9] Guo, Y.-N.; Xu, G.-F.; Gamez, P.; Zhao, L.; Lin, S.-Y.; Deng, R.; Tang J.; Zhang, H.-J., J. Am. Chem. Soc., 2010, 132, 8538-8539.

[10] Guo, Y.-N.; Xu, G.-F.; Guo, Y.; Tang, J., Dalton Trans., 2011, 40, 9953-9963.

[11] Guo, Y.-N.; Xu, G.-F.; Wernsdorfer, W.; Ungur, L.; Guo, Y.; Tang, J.; Zhang, H.-J.; Chibotaru L. F.; Powell, A. K., J. Am. Chem. Soc., 2011, 133, 11948-11951.

[12] Han, T.; Ding, Y.-S.; Li, Z.-H.; Yu, K.-X.; Zhai, Y.-Q.; Chilton, N. F.; Zheng, Y.-Z., Chem. Commun., 2019, 55, 7930-7933.

[13] Jeletic, M.; Lin, P.-H.; Le Roy, J. J.; Korobkov, I.; Gorelsky, S. I.; Murugesu, M., J. Am. Chem. Soc., 2011, 133, 19286-19289.

[14] Habib, F.; Luca, O. R.; Vieru, V.; Shiddiq, M.; Korobkov, I.; Gorelsky, S. I.; Takase, M. K.; Chibotaru, L. F.; Hill, S.; Crabtree, R. H.; Murugesu, M., Angew. Chem. Int. Ed., 2013, 52, 11290-11293. 
[15] Habib, F.; Korobkov, I.; Murugesu, M., Dalton Trans., 2015, 44, 6368-6373.

[16] Ho, L. T. A.; Chibotaru, L. F., Phys. Rev. B, 2016, 94, 104422.

[17] Ho, L. T. A.; Chibotaru, L. F., Phys. Rev. B, 2018, 98, 174418.

[18] Ding, Y.; Han, T.; Zhai, Y.; Reta, D.; Chilton, N. F.; Winpenny, R. E. P.; Zheng, Y., Chem. Eur. J., 2020, 26, 5893-5902.

Received: 05 August 2020

Accepted: 24 September 2020

Published online: 30 September 2020

ORCID ID for authors

Daniel Reta: 0000-0003-0000-9892

Nicholas Chilton: 0000-0002-8604-0171

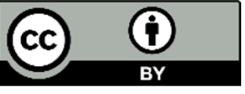

This article is licensed under a Creative Commons Attribution-NonCommercial 4.0 International License, which permits use, sharing, adaptation, distribution and reproduction in any medium or format, as long as it is non-commercial, you give appropriate credit to the original author(s) and the source, provide a link to the Creative Commons license, and indicate if changes were made. The images or other third-party material in this article are included in the article's Creative Commons license, unless indicated otherwise in a credit line to the material. If material is not included in the article's Creative Commons license and your intended use is not permitted by statutory regulation or exceeds the permitted use, you will need to obtain permission directly from the copyright holder. To view a copy of this license, visit http://creativecommons.org/licenses/by/4.0/. 